\title{
Singular Integral Equations in Scattering of Planar Dielectric Waveguide Eigenwaves by the System of Graphene Strips at THz
}

\section{Mstislav E. Kaliberda ( $\square$ KaliberdaME@gmail.com )}

V.N. Karazin Kharkiv National University https://orcid.org/0000-0002-8169-4360

\section{Leonid M. Lytvynenko}

Radioastronomicnij institut Nacional'na akademia nauk Ukraini

\section{Sergey A. Pogarsky}

V N Karazin Kharkiv National University School of Radiophysics Biomedical Electronics and Computer Systems: Harkivs'kij nacional'nij universitet imeni V N Karazina Fakul'tet radiofiziki biomedicnoi elektroniki ta kom'uternih sistem

\section{Research Article}

Keywords: graphene strip, dielectric waveguide, radiation, singular integral equation, leaky-wave antenna

Posted Date: May 28th, 2021

DOl: https://doi.org/10.21203/rs.3.rs-358807/v1

License: (c) (i) This work is licensed under a Creative Commons Attribution 4.0 International License. Read Full License

Version of Record: A version of this preprint was published at Plasmonics on September 30th, 2021. See the published version at https://doi.org/10.1007/s11468-021-01511-9. 
Singular Integral Equations in Scattering of Planar Dielectric Waveguide Eigenwaves by the System of Graphene Strips at $\mathrm{THz}$

Mstislav E. Kaliberda, Leonid M. Lytvynenko, Sergey A. Pogarsky

V.N.Karazin Kharkiv National University, School of Radiophysics, Biomedical Electronics and Computer Systems, Kharkiv, Ukraine

Institute of Radio Astronomy of the National Academy of Sciences of Ukraine, Kharkiv, Ukraine.

Corresponding author: Mstislav E. Kaliberda, e-mail: kaliberdame@gmail.com

\begin{abstract}
We consider the scattering of the H-polarized eigenwaves of a planar dielectric waveguide by a coplanar system of graphene strips in the $\mathrm{THz}$ range. The strips are placed along the centerline of the waveguide. Our treatment is based on the singular integral equations with the Nystrom-type discretization algorithm. Dependences of the scattering characteristics, near and far fields are studied. Frequency scanning radiation pattern are presented. Maximum of the radiated power is observed near the plasmon resonances. The resonant frequency and main lobe level can be controlled by variation of the chemical potential. Applied optimization procedure allows to obtain the radiation pattern with the side-lobe level less than $-20 \mathrm{~dB}$. The presented results can be used in designing of graphene leakywave antennas.
\end{abstract}

Key words: graphene strip, dielectric waveguide, radiation, singular integral equation, leaky-wave antenna.

\title{
1. Introduction
}

Radiating structures based on gratings embedded into a dielectric waveguide transforming eigenwaves to free space waves are promising as low-cost, low-profile, and easy-to-fabricate elements of millimeter-wave devices such as filters or antennas with frequency scanning ability [1,2].

New materials are available today, such as graphene. Graphene can be considered as $2 \mathrm{~d}$ plane of carbon atoms, ideally 1 atom thick. It is famous for being electrically conductive, mechanically strong and optically transparent. Graphene strips can support plasmon polariton surface waves and corresponding plasmon resonances at THz. The resonant frequency can be tuned by applying electrostatic or magnetostatic doping [3, 4]. This feature makes graphene perspective in designing of tunable $\mathrm{THz}$ devices. For example, one can adjust the equivalent impedance of the structure and reduce the reflection. Together with the ability of graphene to absorb the electromagnetic field it becomes very promising element of absorbers $[5,6,7]$. The use of graphene for sensing application is also possible $[8,9,10]$. For the THz range, a numerical analysis of the plasmon resonances on a single graphene strip and corresponding sensing properties in the case of variation of the refractive-index of the host medium is given in [11], and for several strips with substrate is given in [12].

The mutual interaction of graphene strips in the array is smaller than metallic ones because of the short surface plasmon polariton wavelength $[13,14]$. This effect can be used in antennas for reduction of their size. The configuration of tunable leaky-wave antenna with graphene strips embedded into or placed on dielectric slab is discussed in $[15,16,17]$.

Graphene strips may be considered as zero thickness resistive surfaces with the restriction that the strip width is greater than $100 \mathrm{~nm}$. Here one can assume that the edge effect on the graphene conductivity is negligible, and the electrical conductivity model developed for infinite graphene surfaces can be used. For the considered frequency range $1 \ldots 4 \mathrm{THz}$, permittivity and chemical potential values, the spatial dispersion can be neglected [18]. In the absence of spatial dispersion and magnetostatic bias field, the conductivity of graphene is a scalar function $\sigma=\sigma\left(f, \mu_{c}, \tau, T\right)$ of frequency $f$, chemical potential $\mu_{c}$, electron relaxation time $\tau$, and temperature $T$. It can be obtained from the Kubo formalism [19, 20].

Finite-difference time-domain method is one of possible computation instruments for graphene structures [5, 21, 22, 23, 24]. However, as in the case of commercial packages based on the finite-elements method [25], it leads to the huge system of equations due to the large domain of discretization. In addition, the time step is defined by the thickness of graphene which is only one-atom. Another disadvantage is inability to analytically satisfy the radiation conditions, that limits the accuracy of the results to several digits. Approximate techniques, which work much faster, can give reliable results and describe physical effects [17, 26]. 
Another alternative is meshless rigorous techniques such as methods of singular integral equations (SIEs) with Nystrom discretization [27, 28, 29, 30, 31] or method of analytical regularization [32]. They provide a controlled accuracy within a reasonable computation time.

In $[27,28,32]$, several types of resonances are discussed which graphene strip grating embedded into dielectric slab can support. Near the plasmon resonances, an increase in the total scattering and absorption cross section is observed. Except the plasmon resonances which can arise on a single graphene strip, the grating inside the dielectric slab can support the grating-mode resonances. It is shown that in the case of plane wave incidence, near these resonances significant increase in scattering and absorption is also observed.

In the present work we study how the radiation from the dielectric waveguide with finite graphene strip grating can be controlled in the THz range. For solution we use the method of SIEs with discretization based on the Nystrom-type method of discrete singularities [33,34]. Preliminary results were presented in the conference papers $[35,36]$; here results are significantly extended.

\section{Singular Integral Equation}

Let us consider planar dielectric waveguide with coplanar graphene strips placed as shown in Fig.1. We denote the set of strips as $L=\bigcup_{n=1}^{N} L_{n}$, where $L_{n}$ is the $n$th strip. The width of the waveguide is $2 h$, its permittivity is $\varepsilon_{0} \varepsilon$.

We will assume that the field is independent of $x$ and consider only the $H$-polarized waves. Hence the electromagnetic field has the following components: $\left(0, E_{y}, E_{z}\right),\left(H_{x}, 0,0\right)$, which satisfy the Helmholtz equation, the boundary conditions at the dielectric-vacuum interface

$$
H_{x}^{+}=H_{x}^{-}, E_{y}^{+}=E_{y}^{-}, \text {when } z=h \text { or } z=-h,
$$

the boundary conditions on graphene strips and outside the strips,

$$
\begin{aligned}
& E_{y}^{+}=\frac{1}{\sigma}\left(H_{x}^{+}-H_{x}^{-}\right), z=0, y \in L, \\
& H_{x}^{+}=H_{x}^{-}, z=0, y \notin L, \\
& E_{y}^{+}=E_{y}^{-}, z=0,
\end{aligned}
$$

the radiation and the edge conditions. The signs " \pm " indicate the limit values of the field components from above (below) the interfaces.

Outside the dielectric waveguide the radiated field is the field of cylindrical waves which propagate to the infinity. The amplitude of this field decreases as $1 / \sqrt{k \rho}$, when $k \rho \rightarrow \infty$, where $\rho$ is the distance. However, inside the dielectric waveguide, $-h<z<h$, the scattered field can be represented as a sum of the evanescent and propagating eigenwaves. The amplitude of the propagating eigenwaves is non-decreasing function, when $k \rho \rightarrow \infty$. Here we should use the radiation condition in a special form [37, 38].

The incident eigenwave $H^{p}$ of a planar dielectric waveguide with number $p$ propagates from the negative direction of the $y$-axis. The total field can be represented as a sum of the incident and scattered field $H^{\text {total }}=H^{p}+H^{s}$. 
The scattered field can be expanded in terms of Fourier integral in each domain as $H^{s}(y, z)=$

$$
\left\{\begin{array}{l}
\int_{-\infty}^{\infty} a(\xi) \frac{b(\xi / \sqrt{\varepsilon})}{1-g(\xi / \sqrt{\varepsilon})} \exp (i k \xi y+i k \gamma(\xi) z) d \xi, z>h, \\
\int_{-\infty}^{\infty}\left(c(\xi) \exp \left(i k_{1} \xi y-i k_{1} \gamma(\xi) z\right)+\exp \left(i k_{1} \xi y+i k_{1} \gamma(\xi) z\right)\right) \frac{b(\xi)}{1-g(\xi)} d \xi \\
0<z<h, \\
\int_{-\infty}^{\infty}\left(f(\xi) \exp \left(i k_{1} \xi y-i k_{1} \gamma(\xi) z\right)+g(\xi) \exp \left(i k_{1} \xi y+i k_{1} \gamma(\xi) z\right)\right) \frac{b(\xi)}{1-g(\xi)} d \xi \\
-h<z<0, \\
\int_{-\infty}^{\infty} d(\xi) \frac{b(\xi / \sqrt{\varepsilon})}{1-g(\xi / \sqrt{\varepsilon})} \exp (i k \xi y-i k \gamma(\xi) z) d \xi, z<-h,
\end{array}\right.
$$

where $k=\omega \sqrt{\varepsilon_{0} \mu_{0}}, k_{1}=\omega \sqrt{\varepsilon_{0} \varepsilon \mu_{0}}$ are wavenumbers in vacuum and in the dielectric waveguide, $\gamma(\xi)=\sqrt{1-\xi^{2}}$ with non-negative real and imaginary parts,

$$
\begin{aligned}
& a(\xi)=2 \gamma(\xi)(\exp (-i k h \gamma(\xi / \sqrt{\varepsilon}))(\gamma(\xi / \sqrt{\varepsilon}) \sqrt{\varepsilon}+\gamma(\xi)) \\
& +\exp \left(-i h\left(-2 k_{1} \gamma(\xi)+k \gamma(\xi / \sqrt{\varepsilon})\right)\right)(\gamma(\xi / \sqrt{\varepsilon}) \sqrt{\varepsilon}-\gamma(\xi)) / G(\xi) / \sqrt{\varepsilon}, \\
c(\xi)= & \exp \left(i k_{1} h \gamma(\xi)\right)\left(-\gamma^{2}(\xi / \sqrt{\varepsilon}) \varepsilon+\gamma^{2}(\xi)-\exp \left(2 i k_{1} h \gamma(\xi)\right)(\gamma(\xi / \sqrt{\varepsilon}) \sqrt{\varepsilon}-\gamma(\xi))^{2}\right) / G(\xi), \\
& f(\xi)=-(\gamma(\xi / \sqrt{\varepsilon}) \sqrt{\varepsilon}+\gamma(\xi)) \exp \left(-i k_{1} h \gamma(\xi)\right)(\gamma(\xi / \sqrt{\varepsilon}) \sqrt{\varepsilon}+\gamma(\xi)) \\
& \left.+\exp \left(-i h k_{1} \gamma(\xi)\right)(\gamma(\xi / \sqrt{\varepsilon}) \sqrt{\varepsilon}-\gamma(\xi))\right) / G(\xi), \\
& g(\xi)=(\gamma(\xi / \sqrt{\varepsilon}) \sqrt{\varepsilon}-\gamma(\xi)) \exp \left(i k_{1} h \gamma(\xi)\right)(\gamma(\xi / \sqrt{\varepsilon}) \sqrt{\varepsilon}+\gamma(\xi) \\
& \left.+\exp \left(2 i h k_{1} \gamma(\xi)\right)(\gamma(\xi / \sqrt{\varepsilon}) \sqrt{\varepsilon}-\gamma(\xi))\right) / G(\xi), \\
& d(\xi)=-a(\xi), \\
& G(\xi)=\exp \left(i k_{1} h \gamma(\xi)\right)\left(\gamma^{2}(\xi / \sqrt{\varepsilon}) \varepsilon-\gamma^{2}(\xi)+\exp \left(-2 i k_{1} h \gamma(\xi)\right)(\gamma(\xi / \sqrt{\varepsilon}) \sqrt{\varepsilon}+\gamma(\xi))^{2}\right),
\end{aligned}
$$

$b(\xi)$ is unknown function. In fact, (5) is the field expansion in terms of plane waves which propagate in the positive and negative directions of the $z$-axis. In (5), taking into account (6)-(11), the boundary conditions (1), (4) and radiation conditions in the domain $-h<z<h$ are satisfied automatically.

Let us consider the radiation conditions inside the waveguide, $-h<z<h$. Function $1-g(\xi)$ has zeros at the points $\beta_{m}, m=1,2, \ldots$, which correspond to the propagation constants of eigenwaves of dielectric waveguide. For the evanescent waves, points $\beta_{m}$ are complex numbers with $\operatorname{Im} \beta_{m} \neq 0$. For the propagating waves, points $\beta_{m}$ are real numbers, $\operatorname{Im} \beta_{m}=0$. Points $\beta_{m}>0 \quad\left(\beta_{m}<0\right)$ correspond to the waves which propagate to the positive (negative) direction of the $y$-axis. As a result, integrals in (5) has singularities on the integration path. They can be treated as poles. After that the Cauchy integral formula or residue theorem can be used. To calculate these integrals, the integration path should be transformed. First of all, we should note that integrands in (5) are meromorphic functions which satisfy the asymptotic relation

$$
\text { integrand } \sim|\xi|^{-3 / 2} \exp (-k y \operatorname{Im} \xi) \text {, when }|\xi| \rightarrow \infty,|y|>>1 \text {. }
$$

Then, for $y \rightarrow+\infty \quad(y \rightarrow-\infty)$ inside the waveguide, when $-h<z<h$, expression (5) should give us waves that propagate to the positive (negative) direction of the $y$-axis or decay exponentially. The integration over the real axis can be exchanged by the integration over the contour in the form of semicircle in the upper or lower half-space that will enclose our initial integral. From (12) it follows that to obtain convergent integral for $y>0 \quad(y<0)$ we should take semicircle in the upper $\operatorname{Im} \xi>0$ (lower $\operatorname{Im} \xi<0$ ) half-space. For $y>0 \quad(y<0)$, points $\beta_{m}>0$ $\left(\beta_{m}<0\right)$ must be inside the contour and points $\beta_{m}<0\left(\beta_{m}>0\right)$ must be outside the contour. Thus, the integration path should be transformed so it coincides with the real axis everywhere except points $\beta_{m}$. Negative points $\beta_{m}$ 
should be bypassed from above and positive points $\beta_{m}$ should be bypassed from below (see Fig. 2). In this case, the radiation conditions is satisfied.

Finally, the scattered field inside the waveguide, when $y \rightarrow \pm \infty$, is:

$$
\begin{aligned}
& H^{s}(y, z)=-4 \pi \sum_{m=0}^{\infty} \alpha_{m} \sin \left(k_{1} \gamma\left(\beta_{m}\right) z\right) \exp \left(i k\left|\beta_{m} y\right|\right),-h<z<h, \\
& \alpha_{m}=\lim _{\xi \rightarrow \operatorname{sgn}(y) \xi_{m}} b(\xi)\left(\xi-\operatorname{sgn}(y) \xi_{m}\right) .
\end{aligned}
$$

The enforcement of the remaining boundary conditions (2), (3) gives us dual integral equations relatively unknown function $b(\xi)$ :

$$
\begin{aligned}
& \int_{-\infty}^{\infty} b(\xi) \exp \left(i k_{1} \xi y\right) d \xi=0, y \notin L, \\
& i k_{1} \int_{-\infty}^{\infty} \gamma(\xi)(1-c(\xi)) \frac{b(\xi)}{1-g(\xi)} \exp \left(i k_{1} \xi y\right) d \xi \\
& +\frac{2 i k_{1} \sqrt{\varepsilon}}{\sigma Z_{0}} \int_{-\infty}^{\infty} b(\xi) \exp \left(i k_{1} \xi y\right) d \xi=-\frac{\partial}{\partial z} H^{p}(y, 0), \quad y \in L,
\end{aligned}
$$

where $Z_{0}=120 \pi$ Ohm.

The application of Hilbert transform [39] allows us to reduce the dual series equations (14), (15) to SIE over the set of strips

$$
\begin{aligned}
& \frac{1}{\pi} P V \int_{L} \frac{F(\eta)}{\eta-y} d \eta+\frac{1}{\pi} \int_{L} Q(y, \eta) F(\eta) d \eta=-\frac{\partial}{\partial z} H^{p}(y, 0), y \in L . \\
& \frac{1}{\pi} \int_{L_{n}} F(\xi) d \xi=0, n=1,2, \ldots, N,
\end{aligned}
$$

where $Q(y, \eta)$ is the kernel function,

$$
\begin{aligned}
& Q(y, \eta)=k_{1} \int_{0}^{\infty}\left(1+i \frac{\gamma(\xi)}{\xi} \frac{1-c(\xi)}{1-g(\xi)}\right) \sin \left(k_{1} \xi(y-\eta)\right) d \xi \\
& +\left\{\begin{array}{l}
2 i k \pi(\sigma Z)^{-1}, \text { when } \eta \leq y, \\
0, \text { when } \eta>y,
\end{array}\right.
\end{aligned}
$$

$F(y)$ can be expressed in terms of $b(\xi)$ with the help of Fourier transform, $F(y)=i k_{1} \int_{-\infty}^{\infty} \xi b(\xi) \exp \left(i k_{1} \xi y\right) d \xi$.

The first integral in (16) is understood as Cauchy principal value integral. The kernel-function $Q(y, \eta)(18)$ in the second integral in (16) contains poles. The integration contour is shown in Fig.2. However, the integral over infinite semicircle vanishes. Thus, the integration path in the kernel-function coincides with the real axis everywhere except the poles $\beta_{m}$, and poles are bypassed from below. After application of the regularization procedure and Cauchy integral formula, the kernel-function becomes the regular integral. It can be calculated numerically with the use of the quadrature formula such as the Simpson's or Gaussian rules.

For discretization of SIE, the quadrature rule for the Cauchy principal value singular integrals is used [34, 35]. Taking into account the edge condition and representing unknown function as a product of new unknown regular function $u_{n}(t)$ and function with the inverse square root singularity on every strip, $F\left(\psi_{n}(t)\right)=u_{n}(t) / \sqrt{1-t^{2}}$, we can obtain system of SIEs on the standard interval $(-1 ; 1)$. Here $\psi_{n}(t)$ is linear transformation function of $(-1,1)$ to the segment which corresponds to the $n$th strip $L_{n}$. After that, integrands are replaced with interpolating polynomials. The nodes coincide with the zeros of the Chebyshev polynomials of the first kind. The values of $y$, co-called collocation points, are taken from the set of zeros of the Chebyshev polynomials of the second kind. As a result, the set of algebraic equations can be obtained. 


\section{Numerical Results}

\subsection{Convergence}

The solution of (16)-(18) is unique and convergence is based on the theorems [40, 41]. To control the error of numerical results we used the error-function defined as $\operatorname{err}(M)=|S(M)-S(2 M)| /|S(2 M)|$, where $S$ is equal to the radiated power, $M$ is the order of the Chebyshev polynomial. Numerical results are obtained using $\mathrm{C}++$.

Let us consider the system of identical strips of a width $2 d$ placed equidistantly. The period is $l$.

Fig. 3 shows dependences of $\operatorname{err}(M)$. The results are presented for several values of width of strips and frequency. The convergence in monotone and error vanishes, when $M$ becomes larger that certain value. With the normalized width of the strip $k d$ is increased we should take larger value of $M$.

Notice that (12) is valid for $y>>1$. To calculate the near field we regularize integrals in (5) in the same way as in (17) and used composed Gaussian guadrature for integrals without singularities. Firstly, it follows from (12) that values $T^{a m p l}=1-4 \pi \alpha_{1}$ and $R^{a m p l}=-4 \pi \alpha_{-1}$ are reflection and transmission coefficients (amplitude) of the dominant eigenwave of the waveguide. Secondly, amplitude of the field inside the waveguide also equals to the reflection (or transmission) coefficient. Thus,

$$
H^{s}\left(y, z_{0}\right) \rightarrow-4 \pi \alpha_{ \pm 1} \text {, when } y \rightarrow \pm \infty \text {. }
$$

We checked (19) numerically by increasing $|y|$. Eq. (19) is satisfied at the level of machine accuracy. This allows to validate our home-made program block which is connected with the elimination of singularities in (5) and (17).

In $[35,36]$, we demonstrate that results of commercial software HFSS agreed well with our results. At the same time, the radiation patterns calculated with the help of HFSS show slight instability: the width and the angle of the main lobe vary in the interval $\pm 2^{0} \ldots 3^{0}$, the angle and magnitude of the side-lobes significantly depends on the size of the "vacuum box".

\subsection{Scattering Characteristics}

Figs. 4-6 show dependences of the radiation $R a d$, absorption $A$, reflection $R$, and transmission $T$ coefficients (power) as functions of frequency in the case of the dominant eigenwave of dielectric waveguide excitation. The following relation is satisfied: $R a d+A+R+T=1$. The behavior of the curves can be explained in terms of the resonances that the structure under study can support. The maxima of the radiation coefficient correspond to the plasmon resonances (marked as $P_{i}$ ). They depend on the parameters of individual graphene strip. The position of these resonances on the frequency axis can be controlled by variation of the chemical potential. The most effective radiation is observed for $\mu_{c}=1 \mathrm{eV}$. For $\mu_{c}=0.3 \mathrm{eV}$ near the first plasmon resonance, the radiation efficiency is poor, less than $20 \%$. With an increase of the chemical potential, the radiated power also increases near the first plasmon resonance and reaches its maximum value of about $79 \%$ for $\mu_{c}=1 \mathrm{eV}$. For greater number of strips we do not obtain better radiation, when $d=10 \mu \mathrm{m}$. Almost all non-radiated power is absorbed by the graphene strips, so the radiation efficiency is also about $79 \%$. For $\mu_{c}=1 \mathrm{eV}$ near the first plasmon resonance frequency, the reflected and transmitted power does not exceed $2 \%$.

As one can know, the natural waves and corresponding resonances can excite in periodic structures. Except the plasmon resonances, the structure under study can support resonances, which are caused by the periodicity of the displacement of the strips. Such resonances are marked as $N_{q i}$. The position of these resonances mostly depends on the parameters of dielectric slab and the period and is slightly perturbed by the parameters of graphene strips. Thus, it can not be controlled by variation of the chemical potential. To identify these resonances we considered two values of the width of waveguide $h=50 \mu \mathrm{m}$ and $h=70 \mu \mathrm{m}$. These resonances give maxima in the dependences of $R$ and minima in the dependences of $R a d$. In the case of plane wave incidence from domain $z>0$, resonance $N_{12}$ is identified as grating-mode resonance [27, 32]. Near the grating-mode resonances, in extremely narrow frequency band almost total absorption is observed in the infinite graphene grating. These resonances do not arise in the free space. They are also observed in the case of the perfectly electric conducting gratings inside the dielectric slab [42]. The propagation constant $\chi$ of natural waves of periodic structure can be obtained from the following equation [43]:

$$
\operatorname{det}\left(I-\exp (-i \chi l) t e-\operatorname{erer}(I-\exp (i \chi l) e t)^{-1}\right)=0,
$$

where $t$ and $r$ are reflection and transmission matrixes of a single strip, $I$ is the identity matrix, diagonal matrix $e$ has elements $\exp \left(i k_{1} l \beta_{m}\right)$. Fig.7 shows dependences of $\operatorname{Im} \chi$ as a function of period $l$. Extremes of $\operatorname{Im} \chi$ correspond to the resonances of the natural waves of the periodic part of the structure. 


\subsection{Near Field}

Fig.8 shows the total field distribution near the plasmon resonances $P_{i}$. Field has maxima along the strip. The number of maxima is equal to the number of resonance $i$. As a rule, the first plasmon resonance is much more pronounced. One can observe much more pronounced maxima near $P_{1}$ in Figs.4-6 as well as a high field concentration near the graphene strips.

Fig.9 shows the total field distribution near the resonances of the natural waves of periodic part of the structure $N_{q i}$. The field distribution near the resonances $N_{q i}$ significantly differs from that near the plasmon resonances. It is clearly seen that the field near resonances $N_{q i}$ has different number of variations along the $y$ and $z$ axes inside the waveguide. The total field has $q$ maxima of the amplitude on a single period $(l \cdot n ; l \cdot(n+1))$ and $i$ maxima on the interval $0<z<h$.

\subsection{Far Field}

Let us study the far radiated field for various parameters. Fig.10 shows the radiation patterns (power) near the first plasmon resonance $P_{1}$ and near the resonance of natural wave of periodic structure $N_{12}$. The patterns are normalized by the global maximum which corresponds to the first plasmon resonance, when $f=2.46 \mathrm{THz}$, $\mu_{c}=1 \mathrm{eV}$. We take the parameters of the structure so that only one dominant eigenwave of dielectric waveguide can propagate. The results are presented for the first plasmon resonance $P_{1}$ for $\mu_{c}=0.3 \mathrm{eV}$ and $\mu_{c}=1 \mathrm{eV}$ as well as for the resonance $N_{12}$. When the parameters of the structure correspond to the plasmon resonance, amplitude of the main lobe reaches its maximum. The strong dependence of the graphene conductivity on the chemical potential allows to tune the antenna resonant frequency and the main lobe magnitude.

Despite the large reflection near the resonances of natural waves of periodic structure $N_{p i}$, for practical applications resonances with even indexes can be interesting. Near $N_{12}$, in-phase excitation of currents on strips is observed. As a result, the angle of the main lobe of the radiations pattern is $90^{\circ}$. As we mentioned above, the position of resonances $N_{p i}$ does not depend on the parameters of graphene strips. However, the amplitude of the main lobe here can be controlled by variation of the chemical potential.

As it is usual for the radiating periodic structures, the considered structure shows the frequency-scanning ability. The angle of the main lobe mostly depends on the normalized period $k l$. Fig. 11 demonstrates the diffraction patterns with the frequency dependent main lobe angle for the same value of the chemical potential $\mu_{c}=1 \mathrm{eV}$.

The possibility of independent biasing each strip gives additional degrees of freedom. In antennas design it is required that the radiation pattern has the lowest side-lobe level. By variation of the chemical potential of every individual strip in the array we are going to reduce the side-lobe level. The results are presented in Fig.12 and Fig.13 for two values of frequency, $f=2.7 \mathrm{THz}$ and $f=3 \mathrm{THz}$. The value of the strip width and period is the same. Our purpose is to obtain the side-lobe level less that $-20 \mathrm{~dB}$. As seen, the actual side-lobe level is in good agreement with the desired one. The values of the chemical potential are given in Table 1. We used the gradient descent algorithm, 150 iterations. $-20 \mathrm{~dB}$ angular width of the main lobe of the obtained radiation pattern is about $25^{\circ}$ for $f=2.7 \mathrm{THz}$ and about $20^{\circ}$ for $f=3 \mathrm{THz}$. The radiation pattern for constant value of the chemical potential $\mu_{c}=1 \mathrm{eV}$ is also presented for comparison.

\section{Conclusion}

We have studied the scattering of eigenwaves of a planar dielectric waveguide by graphene strip grating in the range from 1 to $4 \mathrm{THz}$. The scheme of solution is based on the mathematically grounded and effective method of singular integral equations with the discretization by the Nystrom-type algorithm. It provides the controllable accuracy of the solution. The calculation time of one curve in Figs. 10-12 is about $10 \mathrm{sec}$.

The behaviour of the scattering characteristics of the structure has clear resonant nature. The radiated power and main lobe magnitude is maximal near the plasmon resonances. Variation of the chemical potential allows to tune the resonant frequency and radiated power. Near the resonance of natural waves of periodic part of the structure, the main lobe is perpendicular, but the reflection is high. Every individual graphene strip in the array can be biased separately. In this way we reduce the side lobe level.

We believe that presented results can be potentially used in designing of leaky-wave graphene antennas. 


\section{Declarations}

Ethical Approval

Not applicable

Consent to Participate

Not applicable

Consent to Publish

Not applicable

Authors' contributions

Mstislav E. Kaliberda carried out the derivation of equations, wrote the program code and performed computations, participated in data analysis; Leonid M. Lytvynenko made the problem statement, participated in the derivation of basic equations; Sergey A. Pogarsky participated in data analysis, critically revised the manuscript and helped draft the manuscript. All authors gave final approval for publication.

Funding

This work was supported by the Ministry of Education and Science of Ukraine, grants 0119U002535, 0117 U004964.

Competing Interests

We have no competing interests.

Availability of data and material (data transparency)

The data that support the findings of this study are available from the corresponding author upon reasonable request

Code availability (software application or custom code)

The home-made code that support the findings of this study are available from the corresponding author upon reasonable request 


\section{Literature}

1. Itoh T (1977) application of gratings in a dielectric waveguide for leaky-wave antennas and band-reject filters (Short Papers). IEEE Trans Microwave Theory Techn 25:1134-1138. https://doi.org/10.1109/tmtt.1977.1129287

2. Kalinichev VI (1996) Radiation behavior of planar double-layer dielectric waveguides combined with a finite metal-strip grating. Microw Opt Technol Lett 11:69-73. https://doi.org/10.1002/(sici)1098-

2760(19960205)11:2<69::aid-mop6>3.0.co;2-m

3. Horng J, Chen C-F, Geng B, et al (2011) Drude conductivity of Dirac fermions in graphene. Phys Rev B 83:. https://doi.org/10.1103/physrevb.83.165113

4. Ju L, Baisong G, Jason H, et al (2011) Graphene plasmonics for tunable terahertz metamaterials. Nature Nanotech 6:630-634. https://doi.org/10.1038/nnano.2011.146

5. Xu N, Chen J, Wang J, Qin X, Shi J (2017) Dispersion HIE-FDTD method for simulating graphene-based absorber. IET Microwaves, Antennas \& Propagation 11:92-97. https://doi.org/10.1049/iet-map.2015.0707

6. Winson D, Choudhury B, Selvakumar N, Barshilia H, Nair RU (2019) design and development of a hybrid broadband radar absorber using metamaterial and graphene. IEEE Trans Antennas Propagat. 67:5446-5452. https://doi.org/10.1109/tap.2019.2907384

7. Mishra R, Sahu A, Panwar R (2019) cascaded graphene frequency selective surface integrated tunable broadband terahertz metamaterial absorber. IEEE Photonics J 11:1-10. https://doi.org/10.1109/jphot.2019.2900402

8. Tong K, Wang Y, Wang F, Sun J, Wu X (2019) Surface plasmon resonance biosensor based on graphene and grating excitation. Appl Opt 58:1824. https://doi.org/10.1364/ao.58.001824

9. Asgari S, Granpayeh N (2019) Tunable mid-infrared refractive index sensor composed of asymmetric double graphene layers. IEEE Sensors J 19:5686-5691. https://doi.org/10.1109/jsen.2019.2906759

10. Dukhopelnykov SV, Sauleau R, Garcia-Vigueras M, Nosich AI (2019) Combined plasmon-resonance and photonic-jet effect in the $\mathrm{THz}$ wave scattering by dielectric rod decorated with graphene strip. Journal of Applied Physics 126:023104. https://doi.org/10.1063/1.5093674

11. Shapoval OV, Nosich AI (2016) Bulk refractive-index sensitivities of the THz-range plasmon resonances on a micro-size graphene strip. J Phys D: Appl Phys 49:055105. https://doi.org/10.1088/0022-3727/49/5/055105

12. Nejat M, Nozhat N (2019) Ultrasensitive THz refractive index sensor based on a controllable perfect MTM absorber. IEEE Sensors J 19:10490-10497. https://doi.org/10.1109/jsen.2019.2931057

13. Ghosh J, Mitra D (2017) Mutual coupling reduction in planar antenna by graphene metasurface for $\mathrm{THz}$ application. Journal of Electromagnetic Waves and Applications 31:2036-2045.

https://doi.org/10.1080/09205071.2016.1277959

14. Zhang B, Jornet JM, Akyildiz IF, Wu ZP (2019) Mutual coupling reduction for ultra-dense multi-band plasmonic nano-antenna arrays using graphene-based frequency selective surface. IEEE Access 7:33214-33225. https://doi.org/10.1109/access.2019.2903493

15. Esquius-Morote M, Gomez-Diaz JS, Perruisseau-Carrier J (2014) Sinusoidally modulated graphene leaky-wave antenna for electronic beamscanning at THz. IEEE Trans THz Sci Technol 4:116-122.

https://doi.org/10.1109/tthz.2013.2294538

16. Fuscaldo W, Burghignoli P, Baccarelli P, Galli A (2016) A reconfigurable substrate-superstrate graphene-based leaky-wave THz antenna. Antennas Wirel Propag Lett 15:1545-1548. https://doi.org/10.1109/lawp.2016.2550198

17. Fuscaldo W, Burghignoli P, Baccarelli P, Galli A (2017) Efficient 2-D leaky-wave antenna configurations based on graphene metasurfaces. Int J Microw Wireless Technol 9:1293-1303.

https://doi.org/10.1017/s1759078717000459 
18. Gomez-Diaz JS, Mosig JR, Perruisseau-Carrier J (2013) Effect of spatial dispersion on surface waves propagating along graphene sheets. IEEE Trans Antennas Propagat 61:3589-3596.

https://doi.org/10.1109/tap.2013.2254443

19. Hanson GW (2008) Dyadic Green's functions and guided surface waves for a surface conductivity model of graphene. Journal of Applied Physics 103:064302. https://doi.org/10.1063/1.2891452

20. Hanson GW (2008) Dyadic Green's functions for an anisotropic, non-local model of biased graphene. IEEE Trans Antennas Propagat. 56:747-757. https://doi.org/10.1109/tap.2008.917005

21. Chen J, Wang J (2016) Three-dimensional dispersive hybrid implicit-explicit finite-difference time-domain method for simulations of graphene. Computer Physics Communications 207:211-216.

https://doi.org/10.1016/j.cpc.2016.06.007

22. Chen J, Xu N, Zhang A, Guo J (2016) Using dispersion HIE-FDTD method to simulate the graphene-based polarizer. IEEE Trans Antennas Propagat 64:3011-3017. https://doi.org/10.1109/tap.2016.2555325

23. Moharrami F, Atlasbaf Z (2020) simulation of multilayer graphene-dielectric metamaterial by implementing sbc model of graphene in the HIE-FDTD method. IEEE Trans Antennas Propagat 68:2238-2245.

https://doi.org/10.1109/tap.2019.2948505

24. Wang X-H, Gao J-Y, Teixeira FL (2019) Stability-improved ADE-FDTD method for wideband modeling of graphene structures. Antennas Wirel Propag Lett 18:212-216. https://doi.org/10.1109/lawp.2018.2886335

25. Tamagnone M, Gómez-Díaz JS, Mosig JR, Perruisseau-Carrier J (2012) Analysis and design of terahertz antennas based on plasmonic resonant graphene sheets. Journal of Applied Physics 112:114915.

https://doi.org/10.1063/1.4768840

26. Slipchenko TM, Nesterov ML, Martin-Moreno L, Nikitin AY (2013) Analytical solution for the diffraction of an electromagnetic wave by a graphene grating. J Opt. 15:114008. https://doi.org/10.1088/2040-8978/15/11/114008

27. Kaliberda ME, Lytvynenko LM, Pogarsky SA (2019) Singular integral equations analysis of THz wave scattering by an infinite graphene strip grating embedded into a grounded dielectric slab. J Opt Soc Am A 36:1787. https://doi.org/10.1364/josaa.36.001787

28. Kaliberda ME, Lytvynenko LM, Pogarsky SA (2020) THz waves scattering by finite graphene strip grating embedded into dielectric slab. IEEE J Quantum Electron 56:1-7. https://doi.org/10.1109/jqe.2019.2950679

29. Ahapova OO, Koshovy GI (2020) On EM wave scattering by coplanar system of flat impedance strips. In 2020 IEEE 40th International Conference on Electronics and Nanotechnology (ELNANO), IEEE. https://doi.org/10.1109/elnano50318.2020.9088752

30. Dukhopelnykov SV, Sauleau R, Nosich AI (2020) integral equation analysis of terahertz backscattering from circular dielectric rod with partial graphene cover. IEEE J Quantum Electron 56:1-8.

https://doi.org/10.1109/jqe.2020.3015482

31. Zinenko TL, Matsushima A, Nosich AI (2020) Terahertz range resonances of metasurface formed by doublelayer grating of microsize graphene strips inside dielectric slab. Proc R Soc A 476:20200173. https://doi.org/10.1098/rspa.2020.0173

32. Zinenko TL, Matsushima A, Nosich AI. 2017 Surface-plasmon, grating-mode, and slab-mode resonances in the $\mathrm{h}$ - and e-polarized thz wave scattering by a graphene strip grating embedded into a dielectric slab. IEEE J Select Topics Quantum Electron 23:1-9. https://doi.org/10.1109/jstqe.2017.2684082

33. Nosich AA, Gandel YV (2007) Numerical analysis of quasioptical multireflector antennas in 2-D with the method of discrete singularities: E-wave case. IEEE Trans Antennas Propagat 55:399-406. https://doi.org/10.1109/tap.2006.889811

34. Gandel' YuV, Dushkin VD (2015) Mathematical model of scattering of polarized waves on impedance strips located on a screened dielectric layer. J Math Sci 212:156-166. https://doi.org/10.1007/s10958-015-2656-2 
35. Kaliberda ME, Pogarsky SA, Kaliberda LM (2020) Modeling of scattering of dielectric waveguide eigenwaves by system of graphene strips at THz. In 2020 IEEE 40th International Conference on Electronics and Nanotechnology (ELNANO), IEEE. https://doi.org/10.1109/elnano50318.2020.9088799

36. Kaliberda ME, Pogarsky SA, Kaliberda LM (2020) Radiation of planar dielectric waveguide eigenwaves scattered by graphene strip grating in THz Range. In 2020 14th European Conference on Antennas and Propagation (EuCAP), IEEE. https://doi.org/10.23919/eucap48036.2020.9135852

37. Andrenko AS, Nosich AI (1992) H-scattering of thin-film modes from periodic gratings of finite extent. Microw. Opt. Technol. Lett. 5:333-337. https://doi.org/10.1002/mop.4650050715

38. Nosich AI (1994) Radiation conditions, limiting absorption principle, and general relations in open waveguide scattering. Journal of Electromagnetic Waves and Applications 8:329-353.

https://doi.org/10.1163/156939394x00902

39. Guillemin EA (1935) Communication network. Vol. 1. John Wiley and Sons Inc, London.

40. Kononenko OS, Gandel YV (2007) Singular and hypersingular integral equations techniques for gyrotron coaxial resonators with a corrugated insert. Int J Infrared Milli Waves 28:267-274. https://doi.org/10.1007/s10762007-9198-8

41. Lifanov IK (1996) Singular integral equations and discrete vortices. VSP, Utrecht.

42. Byelobrov VO, Zinenko TL, Kobayashi K, Nosich AI (2015) Periodicity Matters: Grating or lattice resonances in the scattering by sparse arrays of subwavelength strips and wires. IEEE Antennas Propag Mag. 57:34-45. https://doi.org/10.1109/map.2015.2480083

43. Lytvynenko LM., Prosvirnin SL (2012) Wave Diffraction by Periodic Multilayer Structures, Cambridge Scientific Publishers, Cambridge 
Table 1. Values of the chemical potential of graphene strips.

\begin{tabular}{|l|l|l|}
\hline Strip number & Chemical potential for Fig.12 (eV) & Chemical potential for Fig.13 (eV) \\
\hline 1 & 0.544665 & 0.646117 \\
\hline 2 & 0.579733 & 0.627085 \\
\hline 3 & 0.729025 & 0.865287 \\
\hline 4 & 0.828113 & 0.966635 \\
\hline 5 & 0.893959 & 1.099578 \\
\hline 6 & 0.946739 & 1.029578 \\
\hline 7 & 0.949503 & 1.043876 \\
\hline 8 & 0.972799 & 1.099578 \\
\hline 10 & 0.939843 & 1.046381 \\
\hline & 1.073118 & 0.896205 \\
\hline
\end{tabular}

\section{Figures}

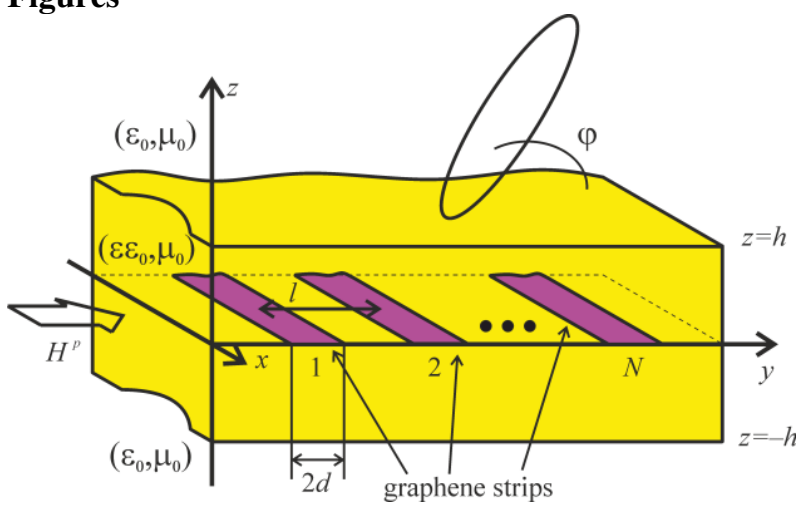

Fig.1 Structure geometry (Created in CorelDRAW X5)

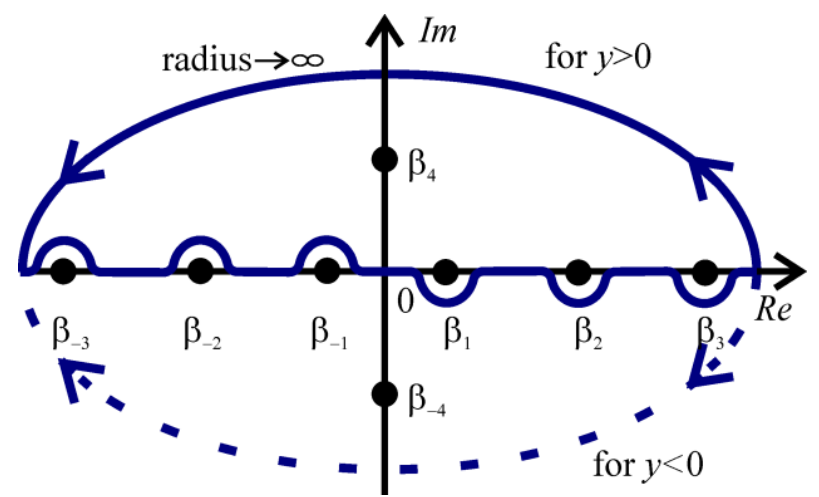

Fig.2 Integration path (Created in CorelDRAW X5)

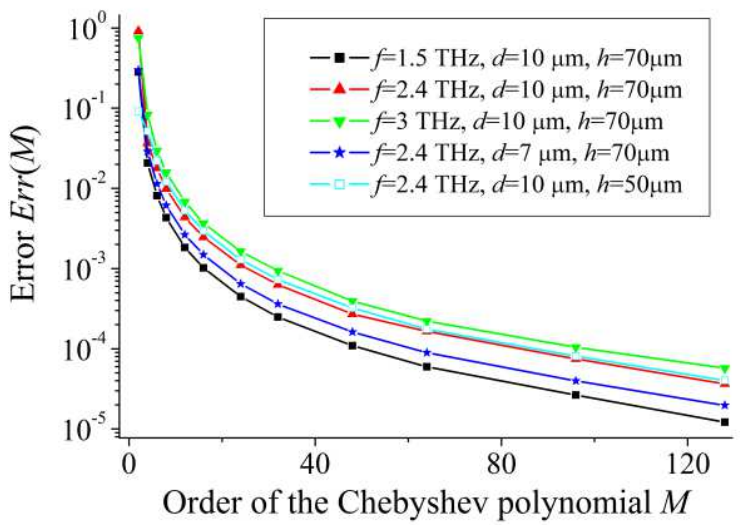

Fig.3 Dependences of the error-function on the order of the Chebyshev polynomial, $\varepsilon=2.25, \mu_{c}=1 \mathrm{eV}$, $T=300 \mathrm{~K}, \tau=1 \mathrm{ps}$ (Created in Origin 6.1) 


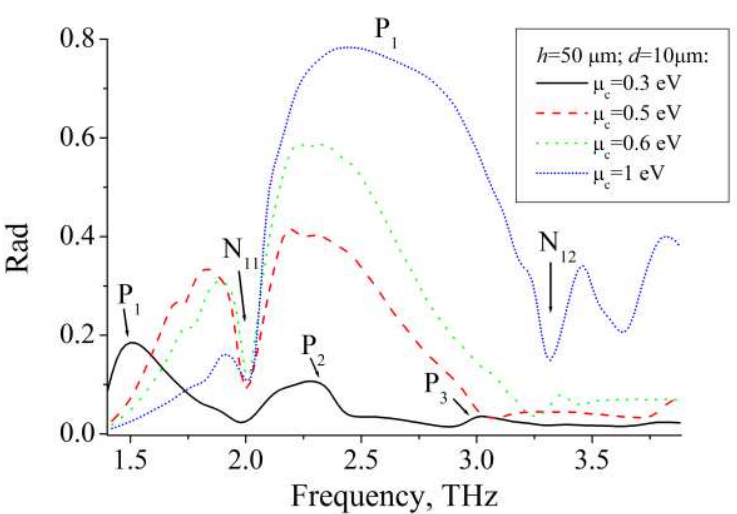

(a)

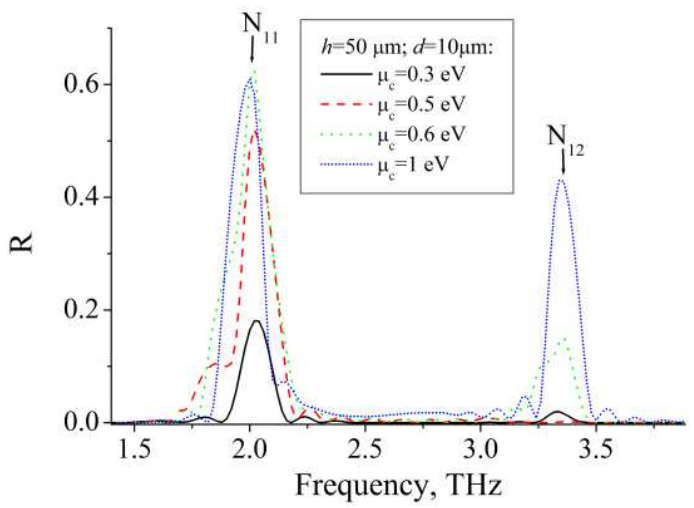

(c)

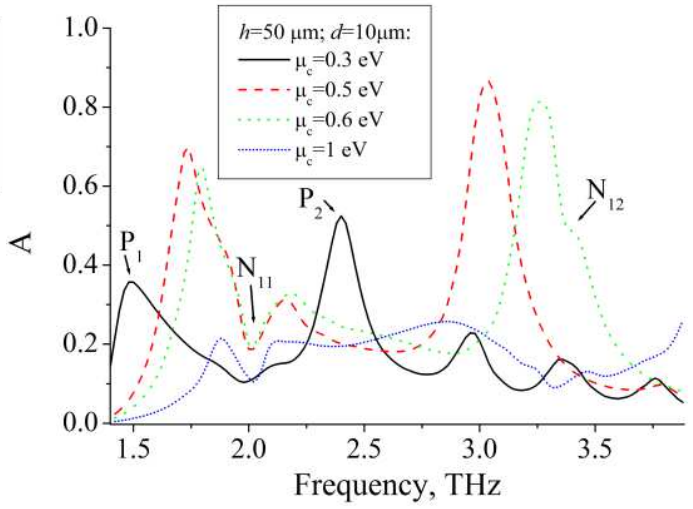

(b)

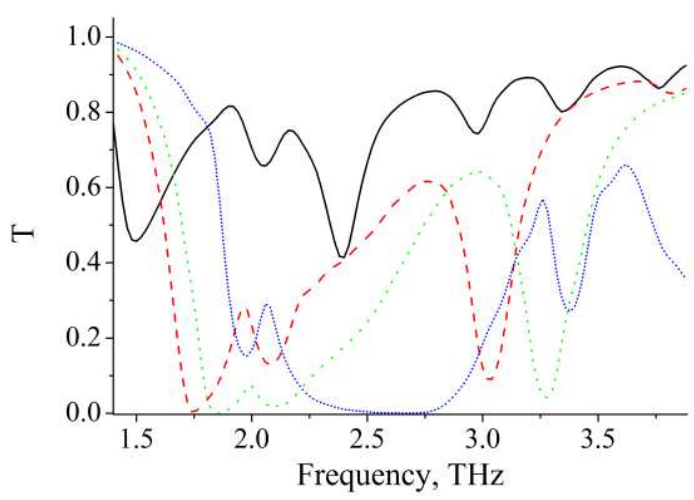

(d)

Fig.4 Dependences of the (a) radiation, (b) absorption, (c) reflection, (d) transmission coefficient on the frequency for $h=50 \mu \mathrm{m}, d=10 \mu \mathrm{m}, \mu_{c}=0.3 \mathrm{eV}$ (solid lines), $\mu_{c}=0.5 \mathrm{eV}$ (dashed lines), $\mu_{c}=0.6 \mathrm{eV}$ (dotted lines), $\mu_{c}=1 \mathrm{eV}$ (shot-dotted lines), $l=70 \mu \mathrm{m}, \varepsilon=2.25, \mu_{c}=1 \mathrm{eV}, T=300 \mathrm{~K}, \tau=1 \mathrm{ps}, N=10$, dominant mode, $p=1$ (Created in Origin 6.1) 


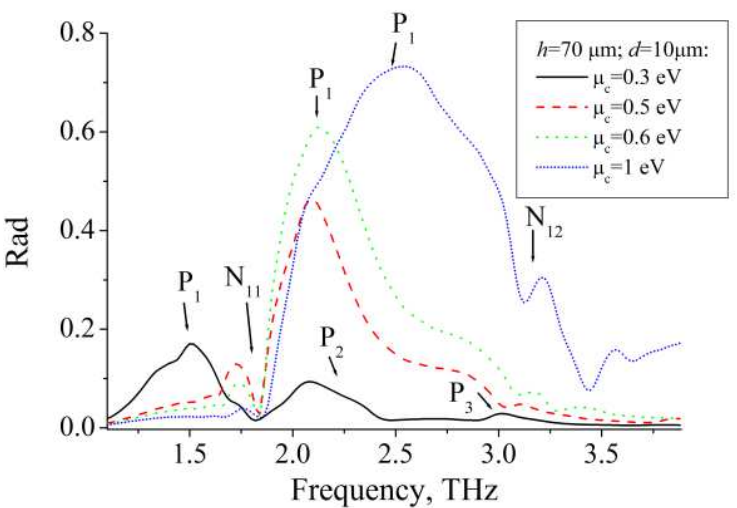

(a)

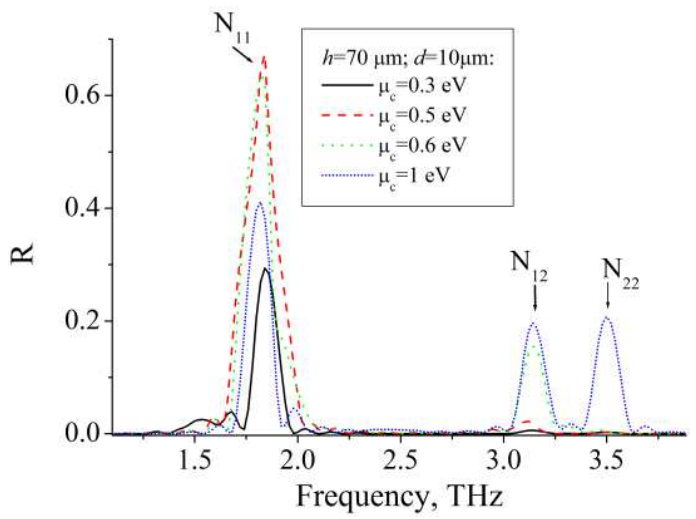

(c)



(b)

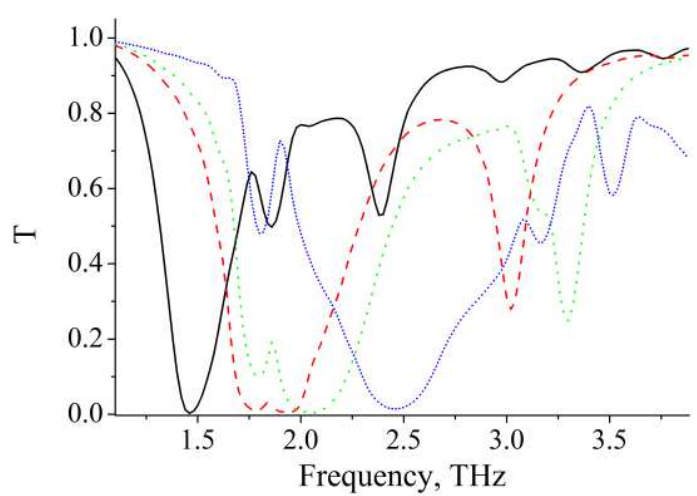

(d)

Fig.5 Dependences of the (a) radiation, (b) absorption, (c) reflection, (d) transmission coefficient on the frequency for $h=70 \mu m, d=10 \mu m$. Other parameters are the same as in Fig. 4 (Created in Origin 6.1)

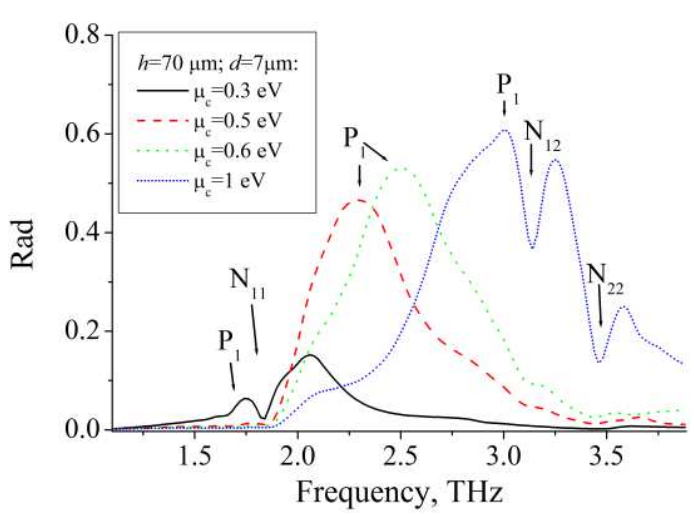

(a)

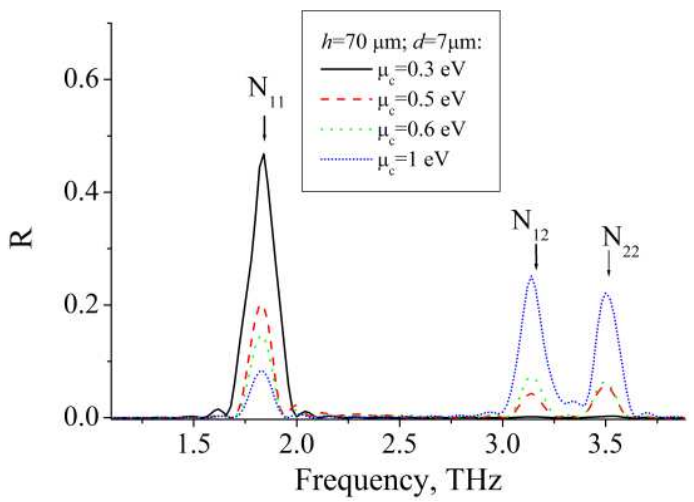

(c)

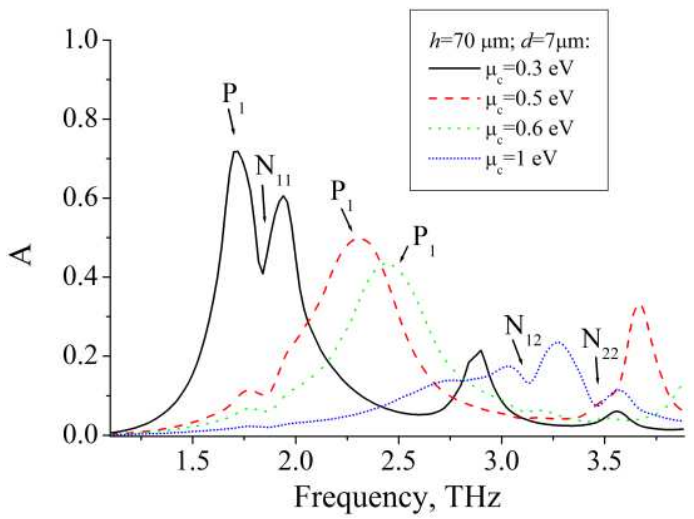

(b)

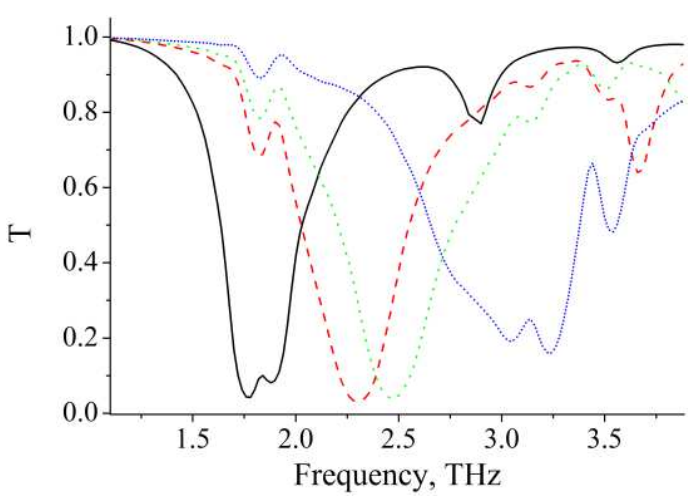

(d)

Fig.6 Dependences of the (a) radiation, (b) absorption, (c) reflection, (d) transmission coefficient on the frequency for $h=70 \mu m, d=7 \mu m$. Other parameters are the same as in Fig. 4 (Created in Origin 6.1) 




Fig.7 Dependences of $\operatorname{Im} \chi$ on the period $l$ for $f=2 \mathrm{THz}$ (solid line), $f=3.34 \mathrm{THz}$ (dashed line), $h=50 \mu m$, $d=10 \mu \mathrm{m}, l=70 \mu \mathrm{m}, \varepsilon=2.25, \mu_{c}=1 \mathrm{eV}, T=300 \mathrm{~K}, \tau=1 \mathrm{ps}, N=10$, dominant mode, $p=1$ (Created in Origin 6.1)



(a)

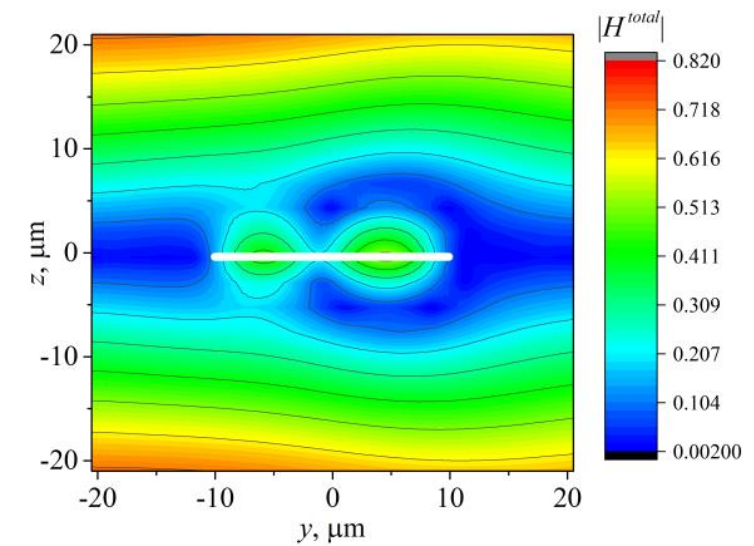

(b)

Fig.8 Field distribution near the plasmon resonances for $h=70 \mu m, d=10 \mu m, l=70 \mu m, \varepsilon=2.25, \mu_{c}=0.3 \mathrm{eV}$, $T=300 \mathrm{~K}, \tau=1 \mathrm{ps}, N=10$, dominant mode, $p=1$. (a) First plasmon resonance, $f=1.5 \mathrm{THz}$; (b) second plasmon resonance, $f=2.5 \mathrm{THz}$ (Created in OriginPro 2015)

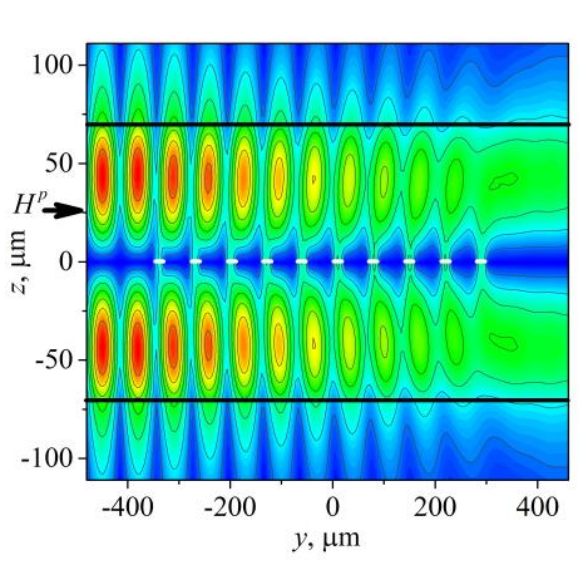

(a)

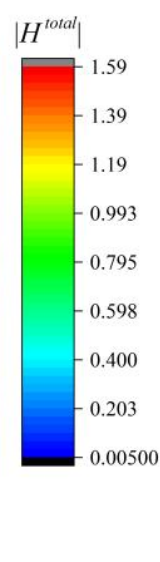

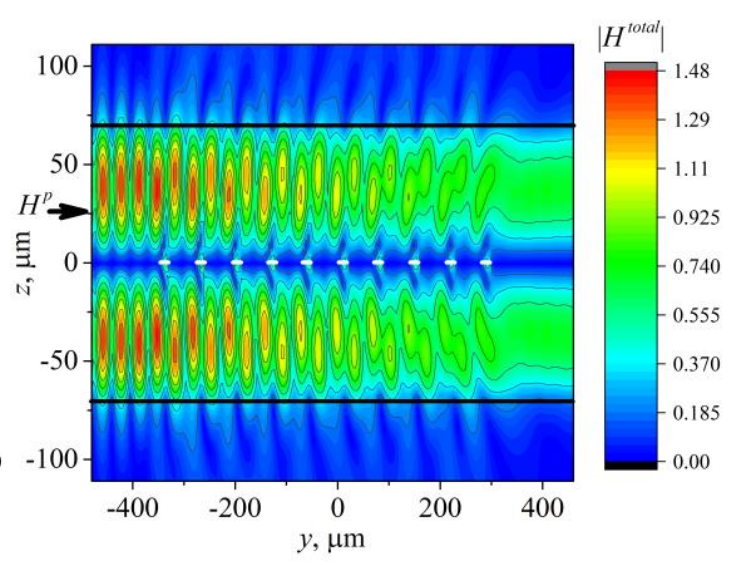

(b) 


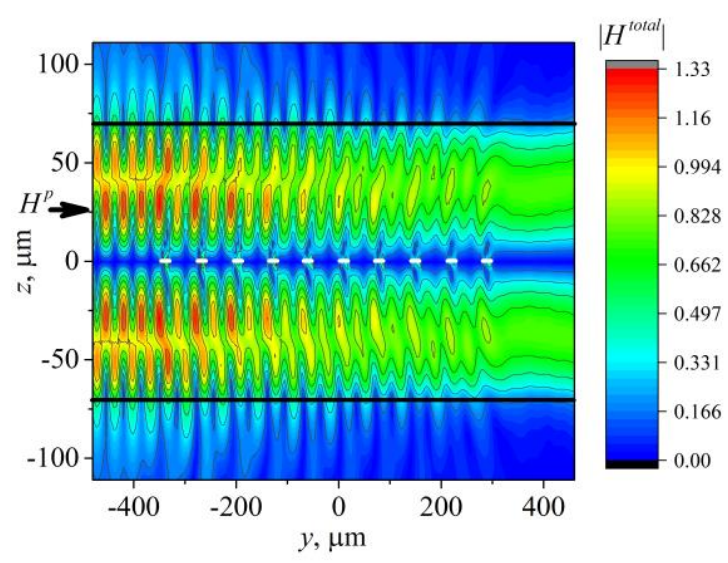

(c)

Fig.9 Field distribution near the resonances of the natural waves of periodic structure for $h=70 \mu m, d=10 \mu m$, $l=70 \mu \mathrm{m}, \varepsilon=2.25, \mu_{c}=1 \mathrm{eV}, T=300 \mathrm{~K}, \tau=1 \mathrm{ps}, N=10$, dominant mode, $p=1$. (a) Resonance $N_{11}$, $f=1.85 \mathrm{THz}$; (b) resonance $N_{12}, f=3.14 \mathrm{THz}$; (c) resonance $N_{22}, f=3.5 \mathrm{THz}$ (Created in OriginPro 2015)

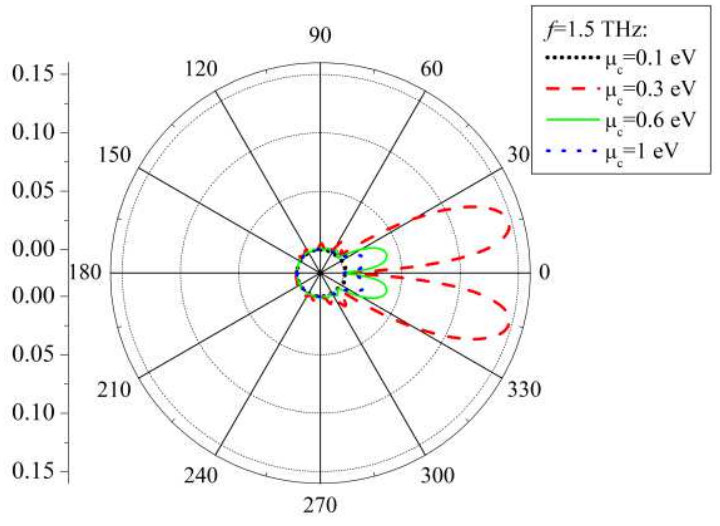

(a)

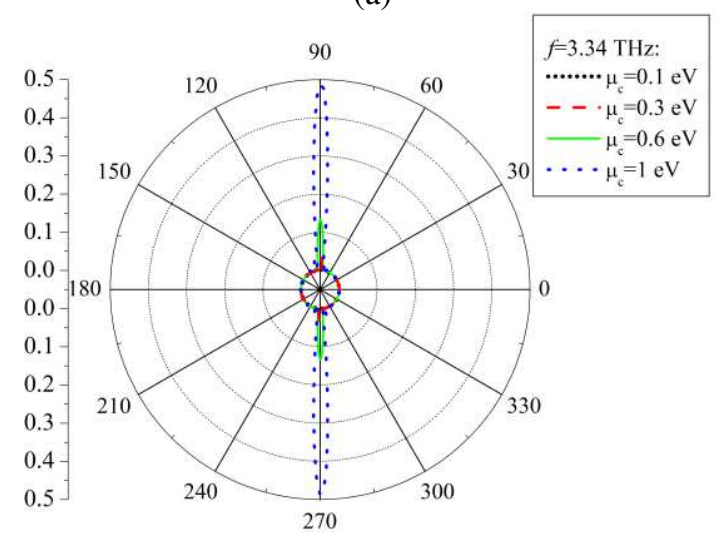

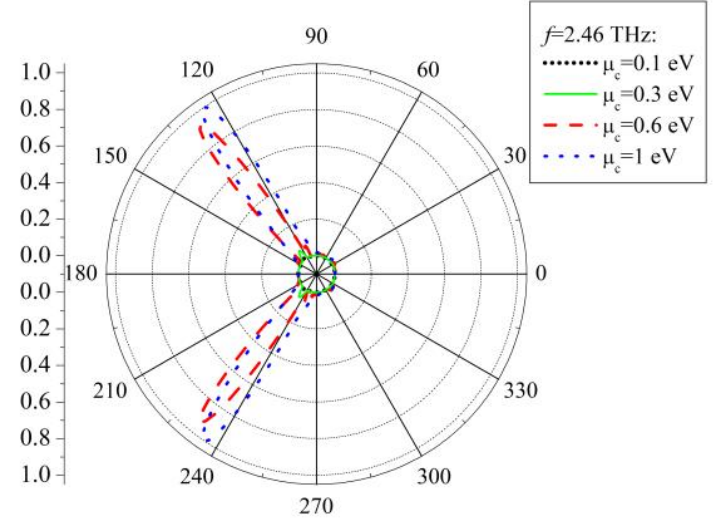

(b)

(c)

Fig.10 Normalized radiation patterns for various values of the chemical potential and frequency, $\mu_{c}=0.1 \mathrm{eV}$ (shotdotted lines), $\mu_{c}=0.3 \mathrm{eV}$ (dashed lines), $\mu_{c}=0.6 \mathrm{eV}$ (solid lines), $\mu_{c}=1 \mathrm{eV}$ (dotted lines), $h=50 \mu m, d=10 \mu m$, $l=70 \mu \mathrm{m}, \varepsilon=2.25, T=300 \mathrm{~K}, \tau=1 \mathrm{ps}, N=10$, dominant mode, $p=1$. (a) Plasmon resonance frequency $f=1.5 \mathrm{THz}$ for $\mu_{c}=0.3 \mathrm{eV}$; (b) plasmon resonance frequency $f=2.46 \mathrm{THz}$ for $\mu_{c}=1 \mathrm{eV}$; (c) $N_{12}$ resonance frequency $f=3.34 \mathrm{THz}$ (Created in OriginPro 2015) 


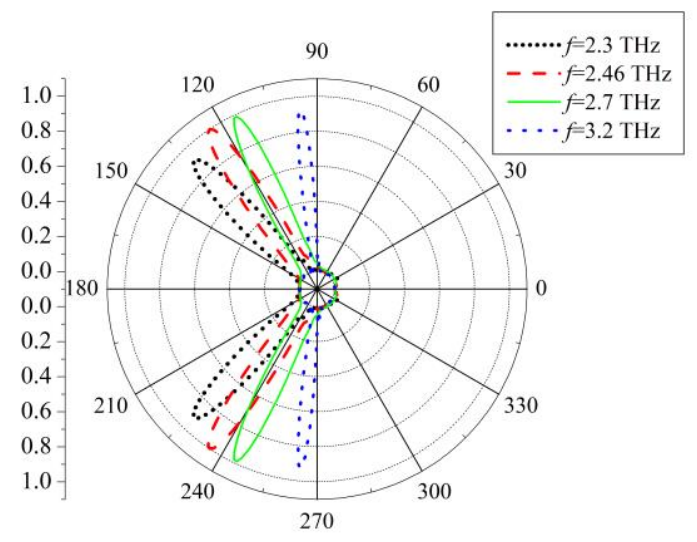

Fig.11 Normalized radiation patterns for various values of the frequency, $f=2.3 \mathrm{THz}$ (shot-dotted lines), $f=2.46 \mathrm{THz}$ (dashed lines), $f=2.7 \mathrm{THz}$ (solid lines), $f=3.2 \mathrm{THz}$ (dotted lines), $\mu_{c}=1, h=50 \mu m, d=10 \mu m$, $l=70 \mu \mathrm{m}, \varepsilon=2.25, T=300 \mathrm{~K}, \tau=1 \mathrm{ps}, N=10$, dominant mode, $p=1$ (Created in OriginPro 2015)

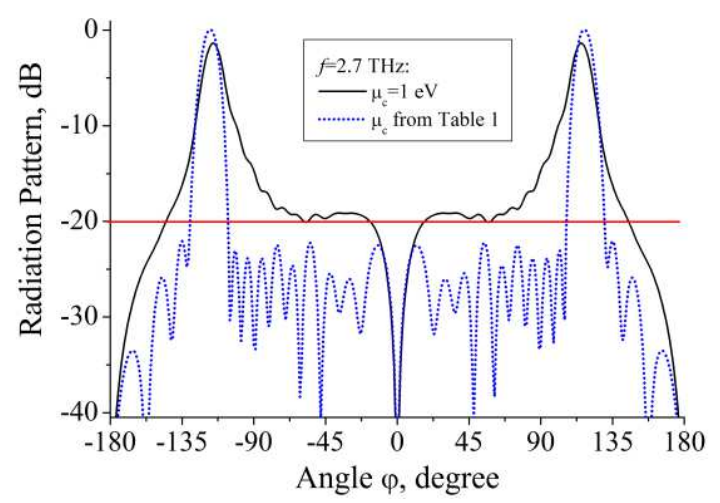

Fig.12 Normalized radiation patterns for constant value of the chemical potential on every strip $\mu_{c}=1 \mathrm{eV}$ (solid line) and for different values of the chemical potential on every strip (dotted line), $f=2.7 \mathrm{THz}, h=50 \mu m$, $d=10 \mu \mathrm{m}, l=70 \mu \mathrm{m}, \varepsilon=2.25, T=300 \mathrm{~K}, \tau=1 \mathrm{ps}, N=10$, dominant mode, $p=1$ (Created in OriginPro 2015)

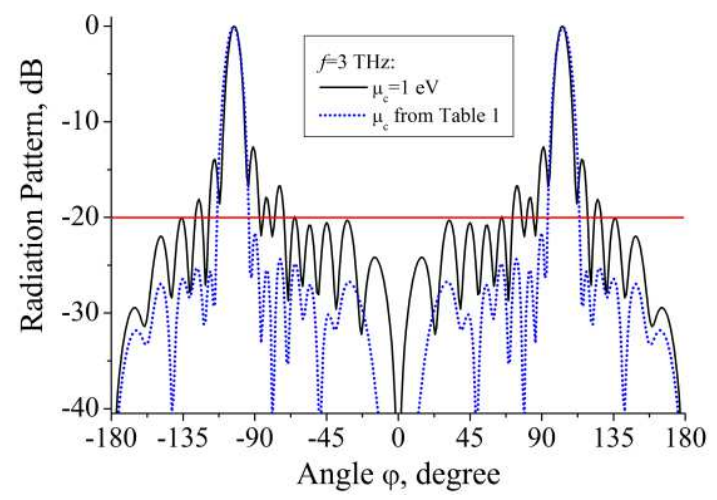

Fig.13 Normalized radiation patterns for constant value of the chemical potential on every strip $\mu_{c}=1 \mathrm{eV}$ (solid line) and for different values of the chemical potential on every strip (dotted line), $f=3 \mathrm{THz}, h=50 \mu m$, $d=10 \mu \mathrm{m}, l=70 \mu \mathrm{m}, \varepsilon=2.25, T=300 \mathrm{~K}, \tau=1 \mathrm{ps}, N=10$, dominant mode, $p=1$ (Created in OriginPro 2015) 